\title{
Quantum and Classical Communication-Space Tradeoffs from Rectangle Bounds
}

\author{
Hartmut Klauck * \\ Institut für Informatik \\ Goethe-Universität Frankfurt \\ 60054 Frankfurt am Main, Germany \\ klauck@thi.informatik.uni-frankfurt.de
}

\begin{abstract}
We derive lower bounds for tradeoffs between the communication $C$ and space $S$ for communicating circuits. The first such bound applies to quantum circuits. If for any problem $f: X \times Y \rightarrow Z$ the multicolor discrepancy of the communication matrix of $f$ is $1 / 2^{d}$, then any bounded error quantum protocol with space $S$, in which Alice receives some $l$ inputs, Bob $r$ inputs, and they compute $f\left(x_{i}, y_{j}\right)$ for the $l \cdot r$ pairs of inputs $\left(x_{i}, y_{j}\right)$ needs communication $C=\Omega(\operatorname{lrd} \log |Z| / S)$. In particular, $n \times n$-matrix multiplication over a finite field $F$ requires $C=\Theta\left(n^{3} \log ^{2}|F| / S\right)$, matrix-vector multiplication $C=\Theta\left(n^{2} \log ^{2}|F| / S\right)$. We then turn to randomized bounded error protocols, and derive the bounds $C=\Omega\left(n^{3} / S^{2}\right)$ for Boolean matrix multiplication and $C=\Omega\left(n^{2} / S^{2}\right)$ for Boolean matrix-vector multiplication, utilizing a new direct product result for the one-sided rectangle lower bound on randomized communication complexity. These results imply a separation between quantum and randomized protocols when compared to quantum bounds in KSW04 and partially answer a question by Beame et al. BTY94.
\end{abstract}

\section{Introduction}

\subsection{Quantum Tradeoffs}

Computational tradeoff results show how spending of one resource must be increased when availability of another resource is limited in solving computational problems. Results of this type have first been established by Cobham Cob66, and have been found to describe nicely the joint behavior of computational resources in many cases. Among the most important such results are time-space tradeoffs, due to the prominence of these two resources. It can be shown that e.g. (classically) sorting $n$ numbers requires that the product of time and space is $\Omega\left(n^{2}\right)$ Bea91, and time $O\left(n^{2} / S\right)$ can also be achieved in a reasonable model of computation for all $\log n \leq S \leq n / \log n$ [PR98.

The importance of such results lies in the fact that they capture the joint behavior of important resources for many interesting problems as well as in the possibility to prove superlinear lower bounds for tradeoffs, while superlinear lower bounds for single computational resources can usually not be obtained with current techniques.

${ }^{*}$ Supported by DFG grant KL 1470/1. Work partially done at Department of Computer Science, University of Calgary, supported by Canada's NSERC and MITACS. 
Quantum computing is an active research area offering interesting possibilities to obtain improved solutions to information processing tasks by employing computing devices based on quantum physics, see e.g. [NC00] for a nice introduction into the field. Since the number of known quantum algorithms is rather small, it is interesting to see which problems might be candidates for quantum speedups. Naturally we may also consider tradeoffs between resources in the quantum case. It is known that e.g. quantum time-space tradeoffs for sorting are quite different from the classical tradeoffs, namely $T^{2} S=\widetilde{\Theta}\left(n^{3}\right)$ KSW04 (for an earlier result see A04]). This shorthand notation is meant as follows: the lower bound says that for all $S$ any algorithm with space $S$ needs time $\widetilde{\Omega}\left(n^{3 / 2} / \sqrt{S}\right)$, while the upper bound says that (in this case for all $\log ^{3} n \leq S \leq n$ ) there is a space $S$ algorithm with time $\widetilde{O}\left(n^{3 / 2} / \sqrt{S}\right)$.

Communication-space tradeoffs can be viewed as a generalization of time-space tradeoffs. Study of these has been initiated in a restricted model by Lam et al. [LTT92, and several tight results in a general model have been given by Beame et al. [BTY94]. In the model they consider two players only restricted by limited workspace communicate to compute a function together. Note that whereas communication-space tradeoffs always imply time-space tradeoffs, the converse is not true: e.g. if players Alice and Bob receive a list of $n$ numbers with $O(\log n)$ bits each, then computing the sorted list of these can be done deterministically with communication $O(n \log n)$ and space $O(\log n)$.

Most of the results in this paper are related to the complexity of matrix multiplication. The foremost question of this kind is of course whether quantum algorithms can break the current barrier of $O\left(n^{2.376}\right)$ for the time-complexity of matrix multiplication [CW90] (it has recently been shown that checking matrix multiplication is actually easier in the quantum case than in the classical case, and can be done in time $O\left(n^{5 / 3}\right)$ [BS04]). In this paper we investigate the communication-space tradeoff complexity of matrix multiplication and matrix-vector multiplication. Communicationspace tradeoffs in the quantum setting have recently been established [KSW04 for Boolean matrixvector product and matrix multiplication. In the former problem there are an $n \times n$ matrix $A$ and a vector $b$ of dimension $n$ (given to Alice resp. to Bob), and the goal is to compute the vector $c=A b$, where $c_{i}=\vee_{j=1}^{n}\left(A[i, j] \wedge b_{j}\right)$. In the latter problem of Boolean matrix multiplication two matrices have to be multiplied with the same type of Boolean product. The paper [KSW04] gives tight lower and upper bounds for these problems, namely $C^{2} S=\widetilde{\Theta}\left(n^{5}\right)$ for Boolean matrix multiplication and $C^{2} S=\widetilde{\Theta}\left(n^{3}\right)$ for Boolean matrix-vector multiplication.

Here we first study these problems in the case when the matrix product is not defined by for the Boolean operations $\wedge$ and $\vee$ (which form a semiring with $\{0,1\}$ ), but over finite fields, and again for quantum circuits. Later we go back to the Boolean product and study the classical complexities of these problems, in order to get a quantum/classical separation for the Boolean case. All these results are collected in the following table. 


\begin{tabular}{|c|c|c|c|c|}
\hline & $\begin{array}{l}\text { Fields } F \\
\text { Matrix Mult. }\end{array}$ & $\begin{array}{l}\text { Fields } F \\
\text { Matrix-Vector } \\
\end{array}$ & $\begin{array}{l}\text { Boolean } \\
\text { Matrix Mult. }\end{array}$ & $\begin{array}{l}\text { Boolean } \\
\text { Matrix-Vector }\end{array}$ \\
\hline $\begin{array}{l}\text { Quantum } \\
\text { upper } \\
\text { bound } \\
\end{array}$ & $\begin{array}{l}O\left(n^{3} \log ^{2}|F| / S\right) \\
\text { obvious }\end{array}$ & $\begin{array}{l}O\left(n^{2} \log ^{2}|F| / S\right) \\
\text { obvious }\end{array}$ & $\begin{array}{l}\widetilde{O}\left(n^{5 / 2} / \sqrt{S}\right) \\
\text { KSW04 }\end{array}$ & $\begin{array}{l}\widetilde{O}\left(n^{3 / 2} / \sqrt{S}\right) \\
\text { KSW04 }\end{array}$ \\
\hline $\begin{array}{l}\text { Quantum } \\
\text { lower } \\
\text { bound }\end{array}$ & $\begin{array}{l}\Omega\left(n^{3} \log ^{2}|F| / S\right) \\
\text { this paper }\end{array}$ & $\begin{array}{l}\Omega\left(n^{2} \log ^{2}|F| / S\right) \\
\text { this paper }\end{array}$ & $\begin{array}{l}\Omega\left(n^{5 / 2} / \sqrt{S}\right) \\
\text { KSW04 }\end{array}$ & $\begin{array}{l}\Omega\left(n^{3 / 2} / \sqrt{S}\right) \\
\text { KSW04 }\end{array}$ \\
\hline $\begin{array}{l}\text { Deterministic } \\
\text { upper } \\
\text { bound }\end{array}$ & $\begin{array}{l}O\left(n^{3} \log ^{2}|F| / S\right) \\
\text { obvious }\end{array}$ & $\begin{array}{l}O\left(n^{2} \log ^{2}|F| / S\right) \\
\text { obvious }\end{array}$ & $\begin{array}{l}O\left(n^{3} / S\right) \\
\text { obvious }\end{array}$ & $\begin{array}{l}O\left(n^{2} / S\right) \\
\text { obvious }\end{array}$ \\
\hline $\begin{array}{l}\text { Randomized } \\
\text { lower } \\
\text { bound }\end{array}$ & $\begin{array}{l}\Omega\left(n^{3} \log ^{2}|F| / S\right) \\
\text { BTY94] }\end{array}$ & $\begin{array}{l}\Omega\left(n^{2} \log ^{2}|F| / S\right) \\
\text { BTY94] }\end{array}$ & $\begin{array}{l}\Omega\left(n^{3} / S^{2}\right) \\
\text { this paper }\end{array}$ & $\begin{array}{l}\Omega\left(n^{2} / S^{2}\right) \\
\text { this paper }\end{array}$ \\
\hline
\end{tabular}

Note that in the above table all upper bounds hold for $\log n \leq S \leq n$, and that the results from BTY94 are actually shown in a slightly different model (branching programs that communicate field elements at unit cost) and hence stated with a factor of $\log |F|$ less there.

\section{$1.2 \quad$ Direct Product Results}

As in KSW04] we use direct product type results to obtain quantum communication-space tradeoff lower bounds for functions with many outputs. In this approach (as in previous proofs concerning such tradeoffs) a space bounded circuit computing a function is decomposed into slices containing a certain amount of communication. Such a circuit slice starts with a (possibly complicated) initial state computed by the gates in previous slices, but this state can be replaced by the totally mixed state at the cost of reducing the success probability by a factor of $1 / 2^{S}$, where $S$ is the space bound. If we manage to show that a circuit with the given resources (but with no initial information) can compute $k$ output bits of the function only with success probability exponentially small in $k$, then $k=O(S)$, and we can prove a tradeoff result by concluding that the number of circuit slices times $O(S)$ must be larger than the number of output bits.

A direct product result says that when solving $k$ instances of a problem simultaneously the success probability will go down exponentially in $k$. There are two different types of direct product results. In a strong direct product result we try to solve $k$ instances with $k$ times the resources that allow us to solve the problem on one instance with probability $2 / 3$. In a weak direct product theorem we have only the same amount of resources as for one instance.

Our approach is to show direct product type results for lower bound techniques that work for quantum resp. randomized communication complexity of functions $f$. We focus on lower bound methods defined in terms of the properties of rectangles in the communication matrix of $f$. There are several techniques available now for proving lower bounds on the quantum communication complexity (see Ra03, Kla01]). The earliest such technique was the discrepancy bound first applied to quantum communication by Kremer Kre95. This bound is also related to the majority nondeterministic communication complexity Kla01.

Definition 1 Let $\nu$ be a distribution on $X \times Y$ and $f$ be any function $f: X \times Y \rightarrow\{0,1\}$. Then let $\operatorname{disc}_{\nu}(f)=\max _{R}\left|\nu\left(R \cap f^{-1}(0)\right)-\nu\left(R \cap f^{-1}(1)\right)\right|$, where $R$ runs over all rectangles in the 
communication matrix of $f$ (see Section [2.2).

In the rest of the paper $\mu$ will always denote the uniform distribution on some domain. disc $(f)$ will be a shorthand for $\operatorname{disc}_{\mu}(f)$. We will also refer to the term maximized above as the discrepancy of a particular rectangle. Since we are dealing with multiple output problems, also a notion of multicolor discrepancy we are going to define later will be useful. $-\log (\operatorname{disc}(f))$ gives a lower bound on the quantum communication complexity [Kre95.

As Shaltiel Sha01 has pointed out, in many cases strong direct product theorems do not hold. He however gives a strong direct product theorem for the discrepancy bound, or rather a XOR-lemma: he shows that

$$
\operatorname{disc}\left(\oplus_{i=1, \ldots, k} f\left(x_{i}\right)\right) \leq \operatorname{disc}(f(x))^{\Omega(k)} .
$$

Previously Parnafes et al. [PRW97] showed a general direct product theorem for classical communication complexity, but in their result the success probability is only shown to go down exponentially in $k / c$, where $c$ is the communication complexity of the problem on one instance, so this result cannot be used for deriving good tradeoff bounds. Klauck et al. KSW04 have recently given a strong direct product theorem for computing $k$ instances of the Disjointness problem in quantum communication complexity.

Instead of the usual direct product formulation ( $k$ independent instances of a problem have to be solved) we first focus on the following setup (a generalized form of matrix multiplication): Alice receives $l$ inputs, Bob receives $r$ inputs, and they want to compute $f\left(x_{i}, y_{j}\right)$ for all $l r$ pairs of inputs for some function $f$. We denote this problem by $f_{l, r}$. We will show that when the communication in a quantum protocol is smaller than the discrepancy bound (for one instance) then the success probability of computing some $k$ of the outputs of $f_{l, r}$ goes down exponentially in $k$ (for all $k$ smaller than the discrepancy bound), and refer to such a result as a bipartite product result. This differs from Shaltiel's direct product result for discrepancy [Sha01 in three ways: first, it only holds when the communication is smaller than the discrepancy bound for one instance (like a weak direct product result), secondly, it deals with correlated input instances (in the described bipartite way). Furthermore it is not about discrepancy of the XOR of the outputs for $k$ instances, but rather about the multicolor discrepancy.

\subsection{Our Results}

The first lower bound result of this paper is the following:

Theorem 1 Let $f: X \times Y \rightarrow\{0,1\}$ with $\operatorname{disc}(f) \leq 1 / 2^{d}$. Then any quantum protocol using space $S$ that computes $f_{l, r}$ needs communication $\Omega(d l r / S)$.

A completely analogous statement can be made for functions $f: X \times Y \rightarrow Z$ for some set $Z$ of size larger than two and multicolor discrepancy, where the lower bound is larger by a factor of $\log |Z|$.

The inner product function over a field $F$ is $I P^{F}(x, y)=\sum_{i=1}^{n} x_{i} \cdot y_{i}$ with operations over $F$. $I P^{G F(2)}$ has been considered frequently in communication complexity theory. It is known that its quantum communication complexity is $\Theta(n)$ (the lower bound can be proved using discrepancy Kre95]). Note that $I P_{n, n}^{F}$ corresponds to the multiplication of two $n \times n$ matrices over $F$, while $I P_{n, 1}^{F}$ is the matrix-vector product. It is well known that $\operatorname{disc}\left(I P^{G F(2)}\right) \leq 2^{-n / 2}$ (see KN97]). 
A generalization of this result given by Mansour et al. MNT93 implies similar bounds on the multicolor discrepancy of inner products over larger fields. Together with a trivial deterministic algorithm in the model of communicating circuits we get the following corollary.

Corollary 1 Assume $\log n \leq S \leq n \log |F|$.

$I P_{n, n}^{F}$ can be computed by a deterministic protocol with space $S$ and communication $O\left(n^{3} \log ^{2}(|F|) / S\right)$, and any bounded error quantum protocol with space $S$ needs communication $\Omega\left(n^{3} \log ^{2}(|F|) / S\right)$ for this problem.

$I P_{n, 1}^{F}$ can be computed by a deterministic protocol with space $S$ and communication $O\left(n^{2} \log ^{2}(|F|) / S\right)$, and any bounded error quantum protocol with space $S$ needs communication $\Omega\left(n^{2} \log ^{2}(|F|) / S\right)$ for this problem.

Using a lemma from MNT93 (also employed in BTY94) we are also able to give a lower bound for pairwise universal hash functions.

Definition 2 A pairwise universal family $Y$ of hash functions from a set $X$ to a set $Z$ has the following properties when $h \in Y$ is chosen uniformly at random:

1. For any $x \in X: h(X)$ is uniformly distributed in $Z$.

2. For any $x, x^{\prime} \in X$ with $x \neq x^{\prime}$, and any $z, z^{\prime} \in Z$, the events $h(x)=z$ and $h\left(x^{\prime}\right)=z^{\prime}$ are independent.

In the problem of evaluating a hash function by a protocol Alice gets $x \in X$, Bob gets a function $h \in Y$, and they compute $h(x)$.

Corollary 2 Any bounded error quantum protocol that evaluates a pairwise universal family of hash functions using space $S$ needs communication at least $\Omega\left(\min \left\{\log (|X|) \cdot \log (|Z|) / S, \log ^{2}(|Z|) / S\right\}\right)$.

Beame et al. BTY94] have established the first term in the above expression as a lower bound for randomized communicating circuits. Hence our quantum lower bound is weaker for hash functions that map to a small domain.

There are many examples of pairwise universal hash function, see MNT93. Let us just mention the function $f: G F(r) \times G F(r)^{2} \rightarrow G F(r)$ defined by $f(x,(a, b))=a \cdot x+b$. If $n=\lceil\log r\rceil$ then this function has a quantum communication tradeoff $C S=\Omega\left(n^{2}\right)$. Also there are universal hash functions that can be reduced to matrix-multiplication and matrix-vector multiplication over finite fields, and we could have deduced the result about matrix-vector multiplication in Corollary 1 from the above result. The result about matrix multiplication would not follow, since the standard reduction from convolution (see [MNT93, matrix multiplication itself is not a hash function) has the problem that for convolution the $\log ^{2}|Z|$ term is much smaller than the $\log |X| \cdot \log |Z|$ term, and we would not get a good lower bound. Also not every function $f_{l, r}$, where $f$ has small discrepancy, is a universal hash function.

We then turn to classical communication-space tradeoffs for Boolean matrix and Boolean matrix-vector multiplication. We show a weak direct product theorem for the one-sided rectangle bound on randomized communication complexity, which allows us to deduce a weak direct product theorem for the classical complexity of the Disjointness problem. Using this we can show a communication-space tradeoff lower bound for Boolean matrix multiplication, a problem posed by Beame et al. BTY94]. 
In the Disjointness problem Alice has an $n$-bit input $x$ and Bob has an $n$-bit input $y$. These $x$ and $y$ represent sets, and $D I S J(x, y)=1$ iff those sets are disjoint. Note that $D I S J$ is $N O R(x \wedge y)$, where $x \wedge y$ is the $n$-bit string obtained by bitwise AND-ing $x$ and $y$. The communication complexity of $D I S J$ has been well studied: it takes $\Theta(n)$ communication in the classical (randomized) world KS92, Ra92] and $\Theta(\sqrt{n})$ in the quantum world BCW98, HW02, AA03, Ra03. A strong direct product theorem for the quantum complexity of Disjointness has been established in [KSW04, but the randomized case was left open. $D I S J_{n, n}$ is (the bitwise negation of) the Boolean matrix product.

Theorem 2 There are constants $\epsilon, \gamma>0$ such that when Alice and Bob have $k \leq \epsilon n$ instances of the Disjointness problem on $n$ bits each, and they perform a classical protocol with communication $\epsilon n$, then the success probability of computing all these instances simultaneously correct is at most $2^{-\gamma k}$.

An application of this gives a classical communication-space tradeoff.

Theorem 3 For the problem DIS $J_{n, n}$ (Boolean matrix multiplication) every randomized space $S$ protocol with bounded error needs communication $\Omega\left(n^{3} / S^{2}\right)$.

For the problem DIS $J_{n, 1}$ (Boolean matrix-vector multiplication) every randomized space $S$ protocol with bounded error needs communication $\Omega\left(n^{2} / S^{2}\right)$.

The proof is in Appendix D. Obvious upper bounds are $O\left(n^{3} / S\right)$ resp. $O\left(n^{2} / S\right)$ for all $\log n \leq$ $S \leq n$. No lower bound was known prior to the recent quantum bounds in KSW04. Note that the known quantum bounds for these problems are tight as mentioned above. For small $S$ we still get near-optimal separation results, e.g. for polylogarithmic space quantum protocols for Boolean matrix multiplication need communication $\widetilde{\Theta}\left(n^{2.5}\right)$, classical protocols $\widetilde{\Theta}\left(n^{3}\right)$. The reason we are able to analyze the quantum situation more satisfactorily is the connection between quantum protocols and polynomials exhibited by Razborov Ra03, allowing algebraic instead of combinatorial arguments.

\section{Definitions and Preliminaries}

\subsection{Communicating Quantum Circuits}

In the model of quantum communication complexity, two players Alice and Bob compute a function $f$ on distributed inputs $x$ and $y$. The complexity measure of interest in this setting is the amount of communication. The players follow some predefined protocol that consists of local unitary operations, and the exchange of qubits. The communication cost of a protocol is the maximal number of qubits exchanged for any input. In the standard model of communication complexity Alice and Bob are computationally unbounded entities, but we are also interested in what happens if they have bounded memory, i.e., they work with a bounded number of qubits. To this end we model Alice and Bob as communicating quantum circuits, following Yao Yao93.

A pair of communicating quantum circuits is actually a single quantum circuit partitioned into two parts. The allowed operations are local unitary operations and access to the inputs that are given by oracles. Alice's part of the circuit may use oracle gates to read single bits from her input, and Bob's part of the circuit may do so for his input. The communication $C$ between the two parties 
is simply the number of wires carrying qubits that cross between the two parts of the circuit. A pair of communicating quantum circuits uses space $S$, if the whole circuit works on $S$ qubits.

In the problems we consider, the number of outputs is much larger than the memory of the players. Therefore we use the following output convention. The player who computes the value of an output sends this value to the other player at a predetermined point in the protocol, who is then allowed to forget the output. In order to make the model as general as possible, we allow the players to do local measurements, and to throw qubits away as well as pick up some fresh qubits. The space requirement only demands that at any given time no more than $S$ qubits are in use in the whole circuit.

For more quantum background we refer to $\mathrm{NC00}$.

\subsection{The Discrepancy Lower Bound and Other Rectangle Bounds}

Definition 3 The communication matrix $M_{f}$ a function $f: X \times Y \rightarrow Z$ with rows and columns corresponding to $X, Y$ is defined by $M_{f}(x, y)=f(x, y)$.

$A$ rectangle is a product set in $X \times Y$. Rectangles are usually labelled, an $\ell$-rectangle being labelled with $\ell \in Z$. $\ell(R)$ gives the label of $R$.

We will make use of the following simple observation.

Proposition 1 Let $R \subseteq X^{l} \times Y^{r}$ be a rectangle. Then the set

$$
R^{\prime}[u, v]=\left\{x_{i}, y_{j} \mid u_{1}, \ldots, u_{i-1}, x_{i}, u_{i+1}, \ldots, u_{l}, v_{1}, \ldots, v_{j-1}, y_{j}, v_{j+1}, \ldots, v_{r} \in R\right\}
$$

is a rectangle in $X \times Y$ for all fixed values $u_{a} \in X$ and $v_{b} \in Y, 1 \leq a, b \leq l, r$.

The discrepancy bound has been defined above. The application of the discrepancy bound to communication complexity is as follows (see Kre95]):

Fact 2 A quantum protocol which computes a function $f: X \times Y \rightarrow\{0,1\}$ correctly with probability $1 / 2+\epsilon$ over a distribution $\nu$ on the inputs (and over its measurements) needs at least $\Omega\left(\log \left(\epsilon /\right.\right.$ disc $\left.\left._{\nu}(f)\right)\right)$ communication.

We will use the following generalization of discrepancy to matrices whose entries have more than two different values.

Definition 4 For a matrix $M$ with $M(x, y) \in Z$ for some finite set $Z$ we define its multicolor discrepancy as

$$
\operatorname{mdisc}(M)=\max _{R} \max _{z \in Z}\left|\left(\mu\left(R \cap f^{-1}(z)\right)-\mu(R) /|Z|\right)\right|,
$$

where the maximization is over all rectangles $R$ in $M$.

The above definition corresponds to the notion of strong multicolor discrepancy used previously in communication complexity theory by Babai et al. BHK01. A matrix with high multicolor discrepancy has rectangles whose measure of one color is very different from the average $\mu(R) /|Z|$. Note that we have defined this only for the uniform distribution $\mu$ here, and that only functions for which all outputs have almost equal probabilities are good candidates for small multicolor discrepancy (e.g. the inner product over finite fields).

We next define the one-sided rectangle bound on randomized communication complexity, see Example 3.22 in [KN97] and also Kla03. 
Definition 5 Let $\nu$ be a distribution on $X \times Y$. Then $\nu$ is (strictly) balanced for $f: X \times Y \rightarrow\{0,1\}$, if $\nu\left(f^{-1}(1)\right)=1 / 2=\nu\left(f^{-1}(0)\right)$.

Definition 6 Let $\operatorname{err}(R, \nu, \ell)=\nu\left(f^{-1}(1-\ell) \mid R\right)$ denote the error of an $\ell$-rectangle $R$. Then let size $(\nu, \epsilon, f, \ell)=\max \{\nu(R): \operatorname{err}(R, \nu, \ell) \leq \epsilon\}$, where $R$ runs over all rectangles in $M_{f}$.

Define bound $\epsilon_{\epsilon}^{(1)}(f)=\max _{\nu} \log (1 /$ size $(\nu, \epsilon, f, 1))$, where $\nu$ runs over all balanced distributions on $X \times Y$. Finally,

$$
\operatorname{bound}(f)=\max \left\{\operatorname{bound}_{1 / 4}^{(1)}(f), \text { bound }_{1 / 4}^{(1)}(\neg f)\right\} .
$$

The application to classical communication is as follows.

Fact 3 For any function $f: X \times Y \rightarrow\{0,1\}$, its (public coin) randomized communication complexity with error $1 / 4$ is lower bounded by bound $(f)$.

\section{Proving Quantum Communication-Space Tradeoffs}

Suppose we are given a communicating quantum circuit that computes $f_{l, r}$, i.e., the Alice circuit gets $l$ inputs from $X$, the Bob circuit gets $r$ inputs from $Y$, and they compute all outputs $f\left(x_{i}, y_{j}\right)$. Furthermore we assume that the output for pair $(i, j)$ is produced at a fixed gate in the circuit.

Our approach to prove the lower bound is by slicing the circuit. Let $\operatorname{mdisc}(f)=1 / 2^{d}$. Then we partition the circuit in the following way. The first slice starts at the beginning, and ends when $d / 100$ qubits have been communicated, i.e., after $d / 100$ qubit wires have crossed between the Alice and Bob circuits. The next slice starts afterwards and also contains $d / 100$ qubits communication and so forth. Note that there are $O(C / d)$ slices, and $l r$ outputs, so an average slice has to make about $\operatorname{lrd} / C$ outputs. We will show that every such slice can produce only $O(S)$ output bits. This implies the desired lower bound.

So we consider what happens at a slice. A slice starts in some state on $S$ qubits that has been computed by the previous part of the computation. Then the two circuits run a protocol with $d / 100$ qubits communication. We have to show that there can be at most $O(S)$ output bits. At this point the following observation will be helpful.

Proposition 4 Suppose there is an algorithm that on input $x$ first receives $S$ qubits of initial information depending arbitrarily on $x$ for free. Suppose the algorithm produces some output correctly with probability $p$.

Then the same algorithm with the initial information replaced by the totally mixed state has success probability at least $p / 2^{S}$.

Suppose the circuit computes the correct output with probability $1 / 2$. Then each circuit slice computes its outputs correctly with probability $1 / 2$. Proposition 4 tells us that we may replace the initial state on $S$ qubits by a totally mixed state, and still compute correctly with probability $(1 / 2) \cdot 1 / 2^{S}$. Hence it suffices to show that any protocol with communication $d / 100$ that attempts to make $\ell$ bits of output has success probability exponentially small in $\ell$. Then $\ell$ must be bounded by $O(S)$. What is left to do is provided by the following bipartite product result.

Theorem 4 Suppose a quantum protocol with communication $d / 100$ makes $k \leq d /(100 \log |Z|)$ outputs for function values $f\left(x_{i}, y_{j}\right)$ of $f: X \times Y \rightarrow Z$ with $\operatorname{mdisc}(f) \leq 2^{-d}$. Then the probability that these outputs are simultaneously correct is at most $(1+o(1)) \cdot|Z|^{-k}$. 
We establish this result in two steps. First we show that for each function with multiple outputs and small multicolor discrepancy all quantum protocols have small success probability.

Lemma 5 If there is a quantum protocol with communication $c$ that computes the outputs of a function $f: X^{l} \times Y^{r} \rightarrow Z^{k}$ so that the success probability of the protocol is $1 /|Z|^{k}+\alpha$ (in the worst case), then $\operatorname{mdisc}(f) \geq \Omega\left(\alpha^{5} / 2^{10 c}\right)$.

Conversely, if $c \leq-\log m \operatorname{disc}(f) / 10-k \log |Z|$, then the success probability of quantum protocols with communication $c$ is at most $(1+o(1)) \cdot|Z|^{-k}$.

The next step is to derive multicolor discrepancy bounds for $f_{l, r}$ from multicolor discrepancy bounds for $f$.

Lemma 6 Let $f: X \times Y \rightarrow Z$ have $\operatorname{mdisc}(f) \leq 2^{-d}$. Let the set $O=\left\{\left(i_{1}, j_{1}\right), \ldots,\left(i_{k}, j_{k}\right)\right\}$ contain the indices of $k$ outputs for $f_{l, r}$. Denote by $f_{O}$ the function that computes these outputs. Then $\operatorname{mdisc}\left(f_{O}\right) \leq O\left(2^{-d / 4}\right)$, if $k \leq d / 5$.

These two lemmas imply Theorem 4 . Their proofs are in Appendix $\mathrm{A}$ resp. Appendix B, Now we can conclude the following more general version of Theorem 1.

Theorem 5 Let $f: X \times Y \rightarrow Z$ with $\operatorname{mdisc}(f) \leq 1 / 2^{d}$. Then every quantum protocol using space $S$ that computes $f_{l, r}$ needs communication $\Omega(d l r \log |Z| / S)$.

Proof. Note that if $S=\Omega(d)$, we are immediately done, since communicating the outputs requires at least $l r \log |Z|$ bits. If $S \leq d / 200$, we can apply Theorem 4 and Proposition 4 Consider a circuit slice with communication $d / 100$ and $\ell$ outputs. Apply Theorem 4 to obtain that the success probability of any protocol without initial information is at most $(1+o(1)) \cdot|Z|^{-k}$ for $k$ being the minimum of $\ell$ and $d /(100 \log |Z|)$. With Proposition 4 we get that this must be at least $(1 / 2) \cdot 2^{-S}$, and hence $k \leq(S+2) / \log |Z|$. In the case $k=d /(100 \log |Z|)$ we get the contradiction $S+2 \geq k \log |Z|=d / 100$ to our assumption, otherwise we get $\ell \leq(S+2) / \log |Z|$ and hence $C /(d / 100) \cdot(S+2) / \log |Z| \geq \operatorname{lr}$ as desired.

We also get the following corollary in the same way.

Corollary 3 Let $f$ be a function with $m$ output bits so that for all $k<d$ and each subset $O$ of $k$ output bits mdisc $\left(f_{O}\right)<2^{-d}$. Then every quantum protocol with communication $C$ and space $S$ satisfies the tradeoff $C S=\Omega(d m)$.

\section{Applications}

In this section we apply Theorem [5] and Corollary [3] to show some explicit communication-space tradeoffs. We have already stated our result regarding matrix and matrix-vector products over finite fields in the introduction (Corollary 1). The only missing piece is an upper bound on the multicolor discrepancy of $I P^{F}$ for finite fields $F$.

Lemma $7 \operatorname{mdisc}\left(\widetilde{I P}^{F}\right) \leq|F|^{-n / 4}$.

Proof. The following is proved in [MNT93]. 
Fact 8 Let $Y$ be a pairwise universal family of hash functions from $X$ to $Z$. Let $A \subseteq X, B \subseteq Y$, and $E \subseteq Z$. Then

$$
\left|\operatorname{Prob}_{x \in A, h \in B}(h(x) \in E)-\frac{|E|}{|Z|}\right| \leq \sqrt{\frac{|Y| \cdot|E|}{|A| \cdot|B| \cdot|Z|}} .
$$

$I P^{F}$ can be changed slightly to give a universal family, with $X=F^{n}$ and $Z=F$, by letting $h(x)=I P^{F}(x, y)+a$ for $y$ drawn randomly from $F^{n}$ and $a$ from $F$. Then the set of hash functions has size $|Y|=|F|^{n+1}$.

To bound the multicolor discrepancy of evaluating the hash family we can set $E$ to contain any single element of $F$. Hence for each rectangle $A \times B$ containing at least $|F|^{(3 / 2) \cdot n}$ entries the right hand side of inequality (II) is at most $|F|^{(n+1) / 2} /\left(\sqrt{|F|^{(3 / 2) \cdot n} \cdot|F|}\right)=|F|^{-n / 4}$. This is an upper bound on $\mu(A \times B)$ times the multicolor discrepancy, and hence also an upper bound on the latter itself. Smaller rectangles can have multicolor discrepancy at most $|F|^{-n / 2-1}$, thus the multicolor discrepancy of evaluating the hash function is at most $|F|^{-n / 4}$. Hence also $I P^{F}$ has small discrepancy: its communication matrix is a rectangle in the communication matrix for the hash evaluation.

Proof of Corollary 2. We again make use of Fact 8] Assume that the output is encoded in binary in some standard way using $\lceil\log |Z|\rceil$ bits. Fix an arbitrary value of $k$ output bits to get a subset $E$ of possible outputs in $Z$. We would like to have $|E| /|Z|=2^{-k}$, but this is not quite possible, e.g. for $Z$ being $\{0, \ldots, p-1\}$ for some prime $p$. If we restrict ourselves to the lower $\log (|Z|) / 2$ bits of the binary encoding of elements of $Z$, however, then each such bit is 1 resp. 0 with probability $1 / 2 \pm 1 / \sqrt{|Z|}$ for a uniformly random $z \in Z$, even conditioned on other bits, so that the probability of a fixed value of $k$ of them is between $(1 / 2-1 / \sqrt{|Z|})^{k}$ and $(1 / 2+1 / \sqrt{|Z|})^{k}$. Then ||$E|/| Z\left|-1 / 2^{k}\right| \leq 2 / \sqrt{|Z|}$.

Let $R=A \times B$ be any rectangle in the communication matrix. Assume that $|R| \geq \sqrt{|X|} \cdot|Y|$. Then the right hand side of (11) is $\leq \sqrt{|Y| /(\sqrt{|X|}|Y|)}=1 /|X|^{1 / 4}$. If $R$ is smaller, then its multicolor discrepancy is at most $1 / \sqrt{|X|}$. So we can apply Corollary 3 with a multicolor discrepancy of at most $|X|^{-1 / 4}+2|Z|^{-1 / 2}$. Note that the number of output bits we consider is $\log |Z| / 2$, and we get $C S=\Omega(\log |X| \cdot \log |Z|)$ or $\Omega\left((\log |Z|)^{2}\right)$, whichever is smaller.

\section{A Direct Product Result for the Rectangle Bound}

Theorem 2 is an immediate consequence of the following direct product result for the rectangle bound, plus a result of Razborov [Ra92].

Lemma 9 Let $f: X \times Y \rightarrow\{0,1\}$ be a function and denote by $f_{k}$ the problem to compute $f$ on $k$ distinct instances. Assume that bound $(f) \geq b$ and that this is achieved on a balanced distribution $\nu$.

Then there is a constant $\gamma>0$ such that the average success probability of each classical protocol with communication $b / 3$ for $f_{k}$ on $\nu^{k}$ is at most $2^{-\gamma k}$ for any $k \leq b$.

The lemma is proved in Appendix C] Now we state the result of Razborov Ra92.

Fact 10 bound $(D I S J) \geq \epsilon n$ for some constant $\epsilon>0$. 
Note that the distribution used in [Ra92 is not strictly balanced, but can be changed to such a distribution easily.

\section{References}

[A04] S. Aaronson. Limitations of Quantum Advice and One-Way Communication. In Proceedings of 19th IEEE Conference on Computational Complexity, pages 320-332, 2004. quant-ph/0402095.

[AA03] S. Aaronson and A. Ambainis. Quantum search of spatial regions. In Proceedings of 44th IEEE FOCS, pages 200-209, 2003. quant-ph/0303041.

[BHK01] L. Babai, T. Hayes, P. Kimmel. The Cost of the Missing Bit: Communication Complexity with Help. Combinatorica, 21(4), pages 455-488, 2001. Earlier version in STOC'98.

[Bea91] P. Beame. A general sequential time-space tradeoff for finding unique elements. SIAM Journal on Computing, 20(2) pages 270-277, 1991. Earlier version in STOC'89.

[BTY94] P. Beame, M. Tompa, and P. Yan. Communication-space tradeoffs for unrestricted protocols. SIAM Journal on Computing, 23(3), pages 652-661, 1994. Earlier version in FOCS'90.

[BCW98] H. Buhrman, R. Cleve, A. Wigderson. Quantum vs. classical communication and computation. 30th ACM Symposium on Theory of Computing, pages 63-68, 1998. quant$\mathrm{ph} / 9802040$.

[BS04] H. Buhrman, R. Špalek. Quantum Verification of Matrix Products. quant-ph/0409035.

[Cob66] A. Cobham. The Recognition Problem for the Set of Perfect Squares. Conference Record of the Seventh Annual Symposium on Switching and Automata Theory ("FOCS"), pages 78-87, 1966.

[CW90] D. Coppersmith, S. Winograd. Matrix Multiplication via Arithmetic Progressions. J. Symb. Comput. 9(3), pages 251-280, 1990.

[HW02] P. Høyer and R. de Wolf. Improved quantum communication complexity bounds for disjointness and equality. In Proceedings of 19th STACS, LNCS 2285, pages 299-310, 2002. quant-ph/0109068.

[KS92] B. Kalyanasundaram and G. Schnitger. The probabilistic communication complexity of set intersection. SIAM Journal on Discrete Mathematics, 5(4), pages 545-557, 1992. Earlier version in Structures'87.

[Kla01] H. Klauck. Lower Bounds for Quantum Communication Complexity. In 42nd IEEE FOCS, pages 288-297, 2001. quant-ph/0106160.

[Kla03] H. Klauck. Rectangle Size Bounds and Threshold Covers in Communication Complexity. In Proceedings of 18th IEEE Conference on Computational Complexity, pages 118-134, 2003. cs.CC/0208006. 
[KSW04] H. Klauck, R. Špalek, R. de Wolf. Quantum and Classical Strong Direct Product Theorems and Optimal Time-Space Tradeoffs. In 45th IEEE FOCS, pages 12-21, 2004. quant-ph/0402123.

[KN97] E. Kushilevitz and N. Nisan. Communication Complexity. Cambridge University Press, 1997.

[Kre95] I. Kremer. Quantum communication. Master's thesis, Hebrew University, Computer Science Department, 1995.

[LTT92] T.W. Lam, P. Tiwari, and M. Tompa. Trade-offs between communication and space. Journal of Computer and Systems Sciences, 45(3), pages 296-315, 1992. Earlier version in STOC'89.

[MNT93] Y. Mansour, N. Nisan, P. Tiwari. The Computational Complexity of Universal Hashing. Theoretical Computer Science, 107(1), pages 121-133, 1993. Earlier version in STOC'90.

[NC00] M. A. Nielsen and I. L. Chuang. Quantum Computation and Quantum Information. Cambridge University Press, 2000.

[PR98] J. Pagter, T. Rauhe. Optimal Time-Space Trade-Offs for Sorting. Proceedings of 39th IEEE FOCS, pages 264-268, 1998.

[PRW97] I. Parnafes, R. Raz, and A. Wigderson. Direct product results and the GCD problem, in old and new communication models. In Proceedings of 29th ACM STOC, pages 363-372, 1997.

[Ra92] A.A. Razborov. On the distributional complexity of disjointness. Theoretical Computer Science, 106(2), pages 385-390, 1992.

[Ra03] A.A. Razborov. Quantum communication complexity of symmetric predicates. Izvestiya of the Russian Academy of Science, Mathematics, 67(1), pages 159-176, 2003. quant$\mathrm{ph} / 0204025$.

[Sha01] R. Shaltiel. Towards proving strong direct product theorems. In Proceedings of 16th IEEE Conference on Computational Complexity, pages 107-119, 2001.

[Yao93] A. C-C. Yao. Quantum circuit complexity. In Proceedings of 34th IEEE FOCS, pages 352-360, 1993.

\section{A Efficient Quantum Protocols and Small Multicolor Discrepancy}

In this section we show Lemma 5. We first need the following fact from Kla01. This result is proved by first decomposing a quantum protocol for each of the possible values of all outputs into few rank one matrices, whose sum expresses the probability of this particular output on the inputs in the communication matrix. Then these matrices are discretized into rectangles (similar to [Yao93, Kre95]). Note that we can assume that the protocol always has a pure global state here, since we have dropped any space restrictions. Also the players do not share any entanglement at the beginning of the protocol, because this is destroyed by replacing the initial state of circuit slices 
by the totally mixed state on $S$ qubits (which in turn can be replaced by $S$ qubits from a pure state on $2 S$ qubits).

Fact 11 Assume there is a quantum protocol with communication $c$ that computes a value of the output $O$ from a finite set $Z$ on each input $x, y$ with probability $p_{x, y}$.

Then for each $\beta \geq 0$ there is a real $w \in[0,1]$, and a set of $O\left(2^{10 c} / \beta^{4}\right)$ rectangles $R(i)$ with weights $w(R(i)) \in\{-w, w\}$, so that

$$
\sum_{i:(x, y) \in R(i)} w(R(i)) \in\left[p_{x, y}-\beta, p_{x, y}\right] .
$$

Hence for our protocol that computes the $k$ outputs of $f$ we can find $|Z|^{k}$ sets $M\left(b_{1}, \ldots, b_{k}\right)$ of rectangles, so that for an input $x, y$ summing the weights of those rectangles in $M\left(b_{1}, \ldots, b_{k}\right)$ that contain $x, y$ gives approximately the probability of output $\left(b_{1}, \ldots, b_{k}\right)$ occurring when the protocol runs on $x, y$. For $R \in M\left(b_{1}, \ldots, b_{k}\right)$ we define $\ell(R)=\left(b_{1}, \ldots, b_{k}\right)$ as its label. Let $M$ be the union of all the $M\left(b_{1}, \ldots, b_{k}\right)$.

Assume the protocol has an advantage of $\alpha$ over a random guess for every input. Set $\beta=\alpha / 2$ when applying the above lemma. For every output value $b_{1}, \ldots, b_{k}$ and for every input $x, y$ such that $f(x, y)=\left(b_{1}, \ldots, b_{k}\right)$ we have

$$
\sum_{R \in M: \ell(R)=\left(b_{1}, \ldots, b_{k}\right),(x, y) \in R} w(R)-1 /|Z|^{k} \geq \alpha-\beta=\alpha / 2
$$

and thus, defining $\delta\left(x, y, b_{1}, \ldots, b_{k}\right)=1-1 /|Z|^{k}$ if $f(x, y)=\left(b_{1}, \ldots, b_{k}\right)$ and $-1 /|Z|^{k}$ otherwise,

$$
\sum_{R \in M:(x, y) \in R} w(R) \delta(x, y, \ell(R)) \geq \alpha / 2
$$

since for all $x, y: \sum_{R \in M:(x, y) \in R} w(R) \leq 1$.

Hence, by averaging,

$$
\sum_{x, y} \mu(x, y) \sum_{R \in M:(x, y) \in R} w(R) \delta(x, y, \ell(R)) \geq \alpha / 2,
$$

and by exchanging sums

$$
\sum_{R \in M} w(R)\left(\mu\left(R \cap f^{-1}(\ell(R))\right)-\mu(R) /|Z|^{k}\right) \geq \alpha / 2 .
$$

Consequently there must be a rectangle $R$ with

$$
\left.\left|\mu\left(R \cap f^{-1}(\ell(R))\right)-\mu(R) /\right| Z\right|^{k} \mid \geq \frac{\alpha}{2} /\left(O\left(2^{10 c} / \beta^{4}\right)=\Omega\left(\alpha^{5} / 2^{10 c}\right) .\right.
$$

This rectangle has the stated multicolor discrepancy. 


\section{B A Bipartite Product Result for Multicolor Discrepancy}

In this section we prove Lemma 6] Suppose that $\operatorname{mdisc}(f) \leq 1 / 2^{d}$. Let $O=\left\{\left(i_{1}, j_{1}\right), \ldots,\left(i_{k}, j_{k}\right)\right\}$ be the set of output labels, i.e., output $(i, j) \in O$ should correspond to $f\left(x_{i}, y_{j}\right)$. Fix some function values $c_{i, j}$ for the elements of $O$, i.e., $f\left(x_{i}, y_{j}\right)=c_{i, j}$. We want to show that each rectangle in $X^{l} \times Y^{r}$ is either very small or contains a fraction of inputs satisfying these constraints that is not much larger than $1 /|Z|^{k}$.

Fix some rectangle $R \subseteq X^{l} \times Y^{r}$. The probability when picking $x, y=x_{1}, \ldots, x_{l}, y_{1}, \ldots y_{r} \in R$ that $f\left(x_{i}, y_{j}\right)=c_{i, j}$ for all $(i, j) \in O$ can be written as the product of conditional probabilities

$$
\prod_{(i, j) \in O} \operatorname{Prob}_{x, y \in R}\left(f\left(x_{i}, y_{j}\right)=c_{i, j} \mid f\left(x_{a}, y_{b}\right)=c_{a, b} \text { for }(a, b)<(i, j) ;(a, b) \in O\right),
$$

assuming some order $<$ on pairs $(i, j)$. For any single term in this product there are three types of conditions: $(a, b)$ may satisfy

1. $a \neq i ; b \neq j$,

2. $a=i ; b \neq j$,

3. $a \neq i ; b=j$.

The first type of condition involves neither $x_{i}$ nor $y_{j}$, the others involve exactly one of them. We can write a term of (2) as

$$
E_{u, v} \operatorname{Prob}_{x, y \in R}\left(f\left(x_{i}, y_{j}\right)=c_{i, j} \mid x_{a}=u_{a}, y_{b}=v_{b} \text { for }(a, b) \neq(i, j), \text { and } \mathcal{C}\right),
$$

where the distribution on $u, v \in X^{l-1} \times Y^{r-1}$ is given by picking $u_{1}, \ldots, u_{l}, v_{1}, \ldots v_{r}$ uniformly from those inputs in $R$ that satisfy $f\left(u_{a}, v_{b}\right)=c_{a, b}$ for all $(a, b)<(i, j) ;(a, b) \in O$, and then dropping $u_{i}, v_{j} . \mathcal{C}$ denotes the conditions of the second and third type.

For all $a, b$ with $a \neq i, b \neq j$ fix any value of $x_{a}=u_{a}$ and $y_{b}=v_{b}$, so that $f\left(x_{a}, y_{b}\right)=c_{a, b}$ if $(a, b)<(i, j)$ and $(a, b) \in O$ (i.e., consider a term in expectation (B3)). Now we are only left with the conditions of the other two types. Also we obtain a rectangle $R^{\prime}[u, v]$ in $X \times Y$, since all inputs but $x_{i}, y_{j}$ are fixed (see Proposition 1).

The second and third types of conditions involve either $x_{i}$ or $y_{j}$. Observe that each such condition $f\left(x_{i}, y_{b}\right)=f\left(x_{i}, v_{b}\right)=c_{i, b}$ partitions $X$ into those $x_{i}$ satisfying it and those who do not, and $f\left(x_{a}, y_{j}\right)=f\left(u_{a}, y_{j}\right)=c_{a, j}$ partitions $Y$. There are at most $k$ such conditions, so all $m$ possible truth values of these conditions partition $X \times Y$ into disjoint rectangles $M_{1}, \ldots, M_{m}$ with $m \leq 2^{k}$. We are interested in the measure of inputs with $f\left(x_{i}, y_{j}\right)=c_{i, j}$ on those $x_{i}, x_{j}$ satisfying the given conditions, i.e., lying in one of these $2^{k}$ rectangles. Hence we need to bound

$$
\operatorname{Prob}_{x, y \in R^{\prime}[u, v]}\left(f(x, y)=c_{i, j} \mid x, y \in M_{\ell}\right)
$$

for one of these rectangles $M_{\ell}$.

Suppose that $\mu\left(R^{\prime}[u, v] \cap M_{\ell}\right) \leq 2^{-d / 2}$. All such rectangles $R^{\prime}[u, v] \cap M_{\ell}$ together contribute at most $k \cdot 2^{k} \cdot 2^{-d / 2}$ to the multicolor discrepancy of $R$, as we argue now. The size of $R$ can be written as follows ( $\mu$ is the uniform distribution on implicit domains). 


$$
\begin{aligned}
\mu(R) & =\sum_{u, v \in R} \mu(u, v) \\
& =\sum_{u_{1}, \ldots, u_{l}, v_{1}, \ldots, v_{r} \in R}\left(\prod_{a \neq i, b \neq j} \mu\left(u_{a}\right) \mu\left(v_{b}\right)\right) \mu\left(u_{i}\right) \mu\left(v_{j}\right) \\
& =E_{u, v} \mu\left(R^{\prime}[u, v]\right) \\
& =\sum_{1 \leq \ell \leq m} E_{u, v} \mu\left(R^{\prime}[u, v] \cap M_{\ell}\right)
\end{aligned}
$$

where the expectation $E_{u, v}$ is over the distribution in which $u_{1}, \ldots, u_{l}, v_{1}, \ldots, v_{r}$ are picked uniformly from $R$, and then $u_{i}$ and $v_{j}$ are dropped.

Hence if we ignore all the small rectangles $R^{\prime}[u, v] \cap M_{\ell}$ in $R$ the measure of ignored inputs is at most $k m 2^{-d / 2}$ : for each of the $k$ terms in the expression (2) and the $m$ possible outputs the above expectation cannot gain more than measure $2^{-d / 2}$ from these rectangles.

So assume that $\mu\left(R^{\prime}[u, v] \cap M_{\ell}\right) \geq 2^{-d / 2}$ always. $R^{\prime}[u, v] \cap M_{\ell}$ is a rectangle in $X \times Y$, and hence has multicolor discrepancy at most $2^{-d}$. Recall that we are interested in

$$
\begin{gathered}
\operatorname{Prob}_{x, y \in R^{\prime}[u, v]}\left(f(x, y)=c_{i, j} \mid x, y \in M_{\ell}\right) \\
=\mu\left(R^{\prime}[u, v] \cap M_{\ell} \cap f^{-1}\left(c_{i, j}\right)\right) / \mu\left(R^{\prime}[u, v] \cap M_{\ell}\right),
\end{gathered}
$$

and so with

$$
\mu\left(R^{\prime}[u, v] \cap M_{\ell} \cap f^{-1}\left(c_{i, j}\right)\right)-\mu\left(R^{\prime}[u, v] \cap M_{\ell}\right) /|Z| \leq 2^{-d},
$$

and $\mu\left(R^{\prime}[u, v] \cap M_{\ell}\right) \geq 2^{-d / 2}$ we get

$$
\operatorname{Prob}_{x, y \in R^{\prime}[u, v]}\left(f(x, y)=c_{i, j} \mid x, y \in M_{\ell}\right) \leq 1 /|Z|+2^{-d / 2} .
$$

So, ignoring small rectangles that altogether contribute at most $k 2^{k} 2^{-d / 2}$ multicolor discrepancy, we have that each term in the product of probabilities (2) is at most $1 /|Z|+2^{-d / 2}$, and hence the product is at most $\left(1 /|Z|+2^{-d / 2}\right)^{k} \leq|Z|^{-k}+2 \cdot 2^{-d / 2}$, since $(1 /|Z|+\gamma)^{k} \leq 1 /|Z|^{k}+2 \gamma$ for all $0 \leq \gamma \leq 1 / 2$ and $|Z| \geq 2$. So the multicolor discrepancy is at most $O\left(k 2^{k} 2^{-d / 2}\right) \leq O\left(2^{-d / 4}\right)$.

\section{A Direct Product Result for the One-Sided Rectangle Bound}

In this section we give the proof of Theorem 2 This theorem is an immediate consequence of our direct product result for the rectangle bound (restated here), plus the aforementioned result of Razborov [Ra92].

Lemma 12 Let $f: X \times Y \rightarrow\{0,1\}$ be a function and denote by $f_{k}$ the problem to compute $f$ on $k$ distinct instances. Assume that bound $(f) \geq b$ and that this is achieved on a balanced distribution $\nu$.

Then there is a constant $\gamma>0$ such that the average success probability of each classical protocol with communication $b / 3$ for $f_{k}$ on $\nu^{k}$ is at most $2^{-\gamma k}$ for any $k \leq b$. 
Proof. Every randomized classical protocol with some success probability $p$ on a fixed distribution can be replaced by a deterministic protocol with the same success probability using standard techniques (since the success probability of a randomized protocol is an expectation over deterministic protocols). So assume we are given a deterministic protocol with communication $c$ and success probability $p$ for $f_{k}$. Such a protocol naturally leads to a partition of $X^{k} \times Y^{k}$ into $2^{c}$ rectangles labelled with the common output of the protocol on the inputs in these rectangles. In our case there are $2^{b / 3}$ rectangles.

Since $\nu$ is (strictly) balanced, on $\nu^{k}$ each sequence of $k$ function values is equally likely. We know that each rectangle on $X \times Y$ that has size at least $1 / 2^{b}$ cannot contain more than a fraction of $3 / 4$ of 1 -inputs to $f$. Intuitively for each sequence of $k$ outputs that has $\Omega(k)$ ones in it, the correctness probability goes down by a factor of $3 / 4$ with each 1-output and is hence exponentially small in $k$.

Assume the following claim:

Claim 13 Each rectangle of size $\geq 2^{-b / 2}$ in $X^{k} \times Y^{k}$ can contain at most a fraction of $2^{-\Omega(k)}$ of inputs having $f_{k}(x)=c$ for every $c \in\{0,1\}^{k}$ with $|c| \in\{k / 3, \ldots, k\}$.

Due to a simple application of the Chernoff bound all but a fraction of $1 / 2^{\Omega(k)}$ of the inputs have function values $c$ with $|c| \geq k / 3$. Then the overall correctness probability of the protocol on $\nu^{k}$ is bounded from above by $1 / 2^{\Omega(k)}$, since apart from the inputs with less than $k / 3$ ones in the function value all other inputs lie in rectangles that either have an error of $1-1 / 2^{\Omega(k)}$ or are smaller than $2^{-b / 2}$. The latter rectangles have a combined measure of at most $2^{b / 3} \cdot 2^{-b / 2} \leq 2^{-\Omega(k)}$, given that $k \leq b$.

Let us prove the claim. Consider a rectangle $R \subseteq X^{k} \times Y^{k}$ of size $2^{-b / 2}$. Fix any output string $c \in\{0,1\}^{k}$ with at least $k / 3$ ones. We are interested in the measure of inputs on $R$ that have $c$ as function value. Again we may write the measure as a product of conditional probabilities

$$
\prod_{i} \operatorname{Prob}\left(f\left(x_{i}, y_{i}\right)=c_{i} \mid f\left(x_{j}, y_{j}\right)=c_{j} \text { for } j<i\right) .
$$

We skip all terms concerning the probability that $f\left(x_{i}, y_{i}\right)=0$. So we are interested in the probability that $f\left(x_{i}, y_{i}\right)=1$ conditioned on $f\left(x_{j}, y_{j}\right)=c_{j}$ for all $j<i$.

Each term may be written as follows.

$$
E_{u, v} \operatorname{Prob}_{x, y \in R}\left(f\left(x_{i}, y_{i}\right)=1 \mid x_{j}=u_{j}, y_{j}=v_{j} \text { for } j \neq i\right),
$$

where the distribution on $u, v \in X^{k-1} \times Y^{k-1}$ is given by picking $u_{1}, \ldots, u_{k}, v_{1}, \ldots, v_{k}$ uniformly from those inputs in $R$ that satisfy $f\left(u_{j}, v_{j}\right)=c_{j}$ for all $j<i$, and then dropping $u_{i}, v_{i}$.

Again fix all $x_{j}, y_{j}, j \neq i$ in any way under the condition that $f\left(x_{j}, y_{j}\right)=c_{j}$ for all $j<i$. This leaves us with a rectangle $R^{\prime}[u, v] \subseteq X \times Y$.

If $\nu\left(R^{\prime}[u, v]\right)<2^{-b}$, then we ignore $R^{\prime}[u, v]$, since all such $R^{\prime}[u, v]$ together will not influence the error of $R$ significantly. More precisely, since all rectangles $R^{\prime}[u, v]$ obtained by fixing $u, v$ are disjoint parts of $R$ when extended by $u, v$ to rectangles in $X^{k} \times Y^{k}$, the combined size of all these small rectangles on $X^{k} \times Y^{k}$ is at most $2^{-b}$. All rectangles ignored in this way in any of the at most $k$ product terms in (4) together have weight at most $k 2^{-b}$, which is at most a $k 2^{-b} / 2^{-b / 2}$ contribution relative to $R$.

So assume that $\nu\left(R^{\prime}[u, v]\right) \geq 2^{-b}$ always. Then clearly $R^{\prime}[u, v]$ contains at most a fraction of $3 / 4$ of 1-inputs to $f$, by the definition of bound $(f)$. All inputs with output $c$ need to have a 1 as output on 
block $i$. Hence the fraction of inputs with output $c$ on $R$ is at most $(3 / 4)^{k / 3}+k \cdot 2^{-b} / 2^{-b / 2}=2^{-\Omega(k)}$, since $k \leq b$.

\section{Classical Communication-Space Tradeoffs}

Proof of Theorem 3. First we prove the lower bound for matrix multiplication. We may assume that $S \leq \gamma \sqrt{\epsilon n} / 2$, for the constants from Theorem 2, because the $n^{2}$ outputs are included in the communication and so otherwise we immediately have $C S^{2}=\Omega\left(n^{3}\right)$. Consider circuit slices of a communicating circuit for matrix multiplication, each circuit slice containing communication $\gamma \epsilon n /(2 S)$. Let $\ell$ denote the number of outputs in any slice. If $\ell<2 S / \gamma$ we will be able to get the lower bound easily. So assume there are more outputs, and choose any $k=2 S / \gamma$ of them. Then we will apply Theorem 2 (details follow later) to show that $k$ outputs can be computed only with success probability $2^{-\gamma k}$, and hence $(1 / 2) \cdot 1 / 2^{S} \leq 2^{-\gamma k}$ with Proposition 4 . This leads to the contradiction that $S+1 \geq 2 S$, hence the slice makes only $2 S / \gamma$ outputs, and so $C \cdot(\gamma \epsilon n)^{-1} \cdot 2 S \cdot 2 S / \gamma \geq n^{2}$.

Consider a classical protocol with $\gamma \epsilon n /(2 S)=\epsilon n / k$ bits of communication. We partition the universe $\{1, \ldots, n\}$ of the Disjointness problems to be computed into $k$ mutually disjoint subsets $U(i, j)$ of size $n / k$, each associated to an output $(i, j)$, which in turn corresponds to a row/column pair $A[i], B[j]$ in the input matrices $A$ and $B$. Assume that there are $a$ outputs $\left(i, j_{1}\right), \ldots,\left(i, j_{a}\right)$ involving $A[i]$. Each output is associated to a subset of the universe $U\left(i, j_{t}\right)$, and we set $A[i]$ to zero on all positions that are not in one of these subsets. Then we proceed analogously with the columns of $B$.

If the protocol computes on these restricted inputs, it has to solve $k$ instances of Disjointness of size $n / k$ each, since $A[i]$ and $B[j]$ contain a single block of size $n / k$ in which both are not set to 0 if and only if $(i, j)$ is one of the $k$ outputs. Hence Theorem 2 is applicable. Note that $k=2 S / \gamma \leq \sqrt{\epsilon n}$ and hence $k \leq \epsilon n / k$ as required.

The proof for matrix-vector product is analogous. 